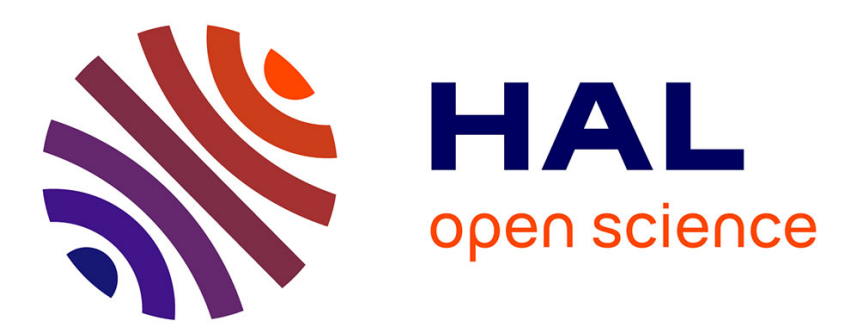

\title{
Quantum electrodynamic effects in semiconductor microcavities - Microlasers and coherent exciton-polariton emission
}

\author{
Y. Yamamoto, F. Matinaga, S. Machida, A. Karlsson, J. Jacobson, G. Björk, \\ T. Mukai
}

\section{To cite this version:}

Y. Yamamoto, F. Matinaga, S. Machida, A. Karlsson, J. Jacobson, et al.. Quantum electrodynamic effects in semiconductor microcavities - Microlasers and coherent exciton-polariton emission. Journal de Physique IV Proceedings, 1993, 03 (C5), pp.C5-39-C5-46. 10.1051/.jp4:1993507 . jpa-00251593

\section{HAL Id: jpa-00251593 https://hal.science/jpa-00251593}

Submitted on 1 Jan 1993

HAL is a multi-disciplinary open access archive for the deposit and dissemination of scientific research documents, whether they are published or not. The documents may come from teaching and research institutions in France or abroad, or from public or private research centers.
L'archive ouverte pluridisciplinaire HAL, est destinée au dépôt et à la diffusion de documents scientifiques de niveau recherche, publiés ou non, émanant des établissements d'enseignement et de recherche français ou étrangers, des laboratoires publics ou privés. 


\title{
Quantum electrodynamic effects in semiconductor microcavities - Microlasers and coherent exciton-polariton emission
}

\author{
Y. YAMAMOTO ${ }^{*, * *}$, F. MATINAGA*, S. MACHIDA* , A. KARLSSON ${ }^{* *}$, J. JACOBSON ${ }^{* *}$, \\ G. BJÖRK ${ }^{* *}$ and T. MUKAI*
}

${ }^{*}$ NTT Basic Research Laboratories, Musashinoshi, Tokyo 180, Japan

${ }^{* *}$ Ginzton Laboratory, Stanford University, Stanford, CA 94305, U.S.A.

\begin{abstract}
We discuss the spontaneous emission of quantum well excitons in a monolithic microcavity. When the quantum well is excited by a nonresonant pump wave at high above the bandgap, the incoherent spontaneous emission is concentrated on the single resonant mode and the laser threshold is reduced by many orders of magnitude. When the quantum well exciton is excited by a resonant pump wave, the coherent spontaneous emission based on a "microcavity exciton-polariton" is observed. The spectral linewidth and the polarization of the pump wave are preserved and the coupling efficiency into the single resonant mode approaches $100 \%$. The microcavity-induced normal mode splitting is observed in the frequency domain by photoexcitation spectrum measurements and in the time domain by pump-probe measurements.
\end{abstract}

\section{Introduction}

Spontaneous emission is not a fixed property of an atom but a consequence of atom-vacuum field coupling. The decay rate, transition energy (frequency), and radiation pattern of spontaneous emission can be altered by modifying the vacuum field fluctuations in the vicinity of the radiating system by a cavity wall. The principle, often referred to as "cavity quantum electrodynamics (cavity QED)", is a classic theoretical problem. A cavity enhanced or suppressed spontaneous decay rate was predicted by Purcell in 1946 [1]. A cavity induced radiative energy shift was predicted by Casimir and Polder in 1948 [2]. A coherent coupling of an atom and single-mode vacuum field was formulated by Jaynes and Cummings in 1963 [3]. Various coherent cavity QED effects such as reversible (or coherent) spontaneous emission, normal mode (or vacuum Rabi) splitting, and quantum collapse and revival were predicted using the Jaynes-Cummings model [4]. Those effects have been observed recently using either Rydberg atoms in microwave superconductor cavities or atoms in optical dielectric cavities [5,6]. The spontaneous emission of quantum well (QW) excitons was also modified by a monolithic microcavity [7]. Recently, the normal mode splitting was observed for the coupled exciton and photon system in a microcavity [8].

The normal modes in solids involving excitons and photons are "polaritons" [9], which are "stationary" (do not radiatively decay) in a bulk material without translational-invariance breaking defects. However, for the excitons confined in a QW which inherently breaks full translational invariance, the electromagnetic decay channel is open for excitons with the in-plane wavenumber $k_{\|}$ lying below the crossing with the photon dispersion line $k_{0}=\left(\omega / c_{0}\right) n$. On the other hand, the QW 
excitons that lie above the crossing with the photon line are quasistationary. For an optically excited 1 s heavy hole exciton by a resonant wave with $k_{\|} \ll k_{0}$, the spontaneous decay times of $\tau_{\text {rad }} \sim 20$ psec were predicted [10]. Although short lifetimes on the order of $24 \mathrm{ps}$ have been reported for a high-quality GaAs/AlAs quantum well [11], generally the observed lifetimes are much longer.

The proposed argument for the long lifetimes, is that the optically excited cold excitons with $k_{\|} \ll k_{0}$ thermalize fairy quickly, for instance, in $\sim 50$ psec at $10 \mathrm{~K}$ and less than 10 psec at 35 $\mathrm{K}$ so that only a small fraction of the thermal distribution of excitons lie below the crossing with the photon line and can decay radiatively. If the exciton population obeys a thermal equilibrium distribution, then the decay time is calculated to be much longer, approximated by $\tau_{\text {rad }} \approx 40 T$ psec where $T$ is a temperature in Kelvin $(5<T<100)$ [10]. The optically excited cold excitons with $k_{\|} \ll k_{0}$ also undergo scattering among themselves and with phonons to lose their initial phase. The free exciton dephasing time is a few psec even at $T<5 \mathrm{~K}$ which is shorter than the above mentioned radiative decay time. Therefore, the phase coherence of the pump wave is not usually preserved in the excitonic spontaneous emission except coherent elastic (Rayleigh) scattering light [12][13].

The situation would be completely different if the QW exciton is placed at an anti-node position of a high- $Q$ microcavity as shown in Fig. 1. The vacuum field fluctuation intensity is enhanced by a factor of $4 /(1-R) \simeq 400$ for exact normal direction $\left(k_{\|}=0\right)$ and at exact resonance $\left(\lambda_{\text {exciton }}=\lambda_{\text {cavity }}\right)$. Since the optically excited cold excitons with $k_{\|} \approx 0$ would radiate mainly into a normal direction, this radiative decay rate would be optimistically enhanced by a similar factor by the microcavity. This means that the cold excitons can radiate before they dephase, thermalize and depolarize, which would lead to the coherent spontaneous emission with a high quantum efficiency close to $100 \%$. This would be a resonant Rayleigh scattering in free space. Since the radiation is reflected back by the cavity mirror and excites the cold excitons again, such folding coherent exciton-photon coupling features a Rabi oscillation, which would resemble the bulk polariton propagation, as shown in Fig. 2.

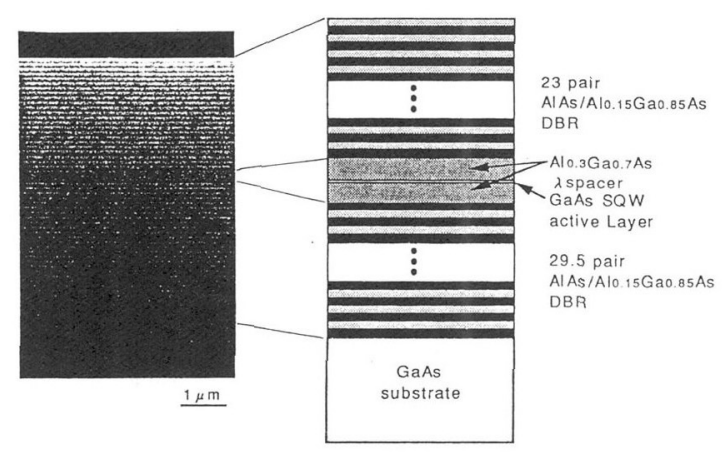

Fig. 1. Vertical structure of the planar microlaser. Active medium is a GaAs quantum well; the $\mathrm{Al}_{0.15} \mathrm{Ga}_{0.85} \mathrm{As} / \mathrm{AlAs}$ Bragg reflectors are spaced by a $\mathrm{Al}_{0.30} \mathrm{Ga}_{0.70} \mathrm{As}$ layer such that a one wavelength cavity is formed.

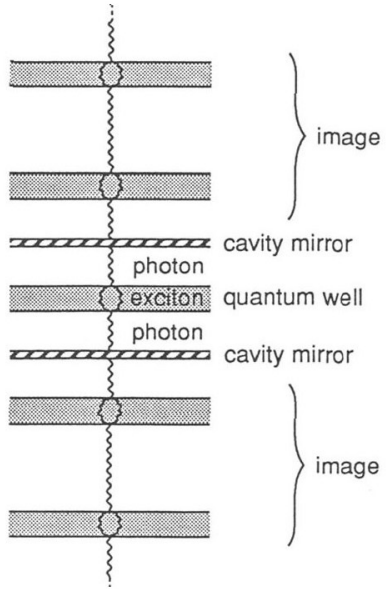

Fig. 2. An exciton-polariton picture in a quantum well high-Q microcavity.

\section{Incoherent spontaneous emission and lasing character- istics}

When the QW is optically excited at well above the bandgap, photoexcited electrons and holes thermalize to the band edge to form thermal equilibrium excitons. In the thermalization process, the phase and polarization of the electron-hole pair is randomized. We will discuss incoherent spontaneous emission from such thermal equilibrium excitons and also lasing characteristic in this section. 
The spontaneous emission coupling efficiency $\beta$ is defined as the ratio between the spontaneous emission radiated into the single cavity resonant mode and the total spontaneous emission. It is important to realize that $\beta$ is the result of the interaction between the cavity and the radiating system. The same cavity will in general have different spontaneous emission coupling efficiency depending on the radiating system.

In Fig. 3 the spontaneous emission per unit solid angle and unit wavelength has been drawn, assuming a Gaussian gain envelope function of the radiating system with a FWHM of $\Delta \lambda_{\text {emission }}=2$ $\mathrm{nm}$, slightly smaller than the cold cavity linewidth of $\Delta \lambda_{\text {cavity }}=2.2 \mathrm{~nm}$ [14]. It is assumed that the dipole moment is oriented in the $x y$-plane and that the emission linewidth $\Delta \lambda_{\text {emission }}$ is homogeneously broadened. The spontaneous emission coupling efficiency can be calculated by integrating the spontaneous emission into the solid angle of the single polarization mode and over the cold cavity bandwidth and dividing it by the same integral over both polarizations, all solid angles and all wavelengths. Although the spontaneous emission per unit solid angle is much larger at the resonance in the normal direction than that in the passband between $\theta=20^{\circ}$ and $\theta=60^{\circ}$, very little solid angle is available in the normal direction. It turns out that the spontaneous emission in the passband carries away most of the emitted spontaneous radiation. In Fig. 4 the spontaneous emission coupling efficiency $\beta$ is drawn as a function of the linewidth ratio $\Delta \lambda_{\text {emission }} / \Delta \lambda_{\text {cavity. }}$. It can be shown that the optimum $\beta$, which is obtained in the limit of $\Delta \lambda_{\text {emission }} / \Delta \lambda_{\text {cavity }} \ll 1$, is independent of the number of DBR pairs and only depends on the refractive-indices of the DBR [15]. If $\Delta \lambda_{\text {emission }}$ is larger than $\Delta \lambda_{\text {cavity }}, \beta$ decreases linearly with $\Delta \lambda_{\text {emission }}$ as manifested by Fig. 4.

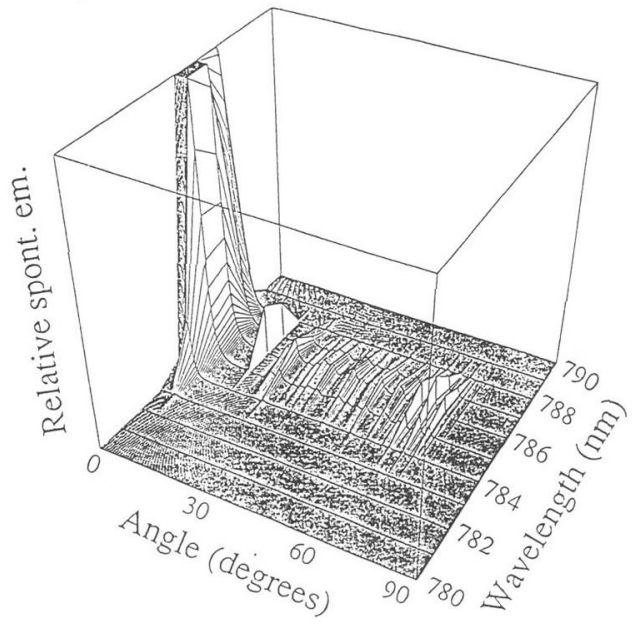

Fig. 3. The computed spontaneous emission per unit solid angle and unit wavelength as a function of angle (in the cavity) and wavelength is shown. The gain linewidth has been assumed to be $2 \mathrm{~nm}$, slightly smaller than the cold cavity linewidth of $2.2 \mathrm{~nm}$.

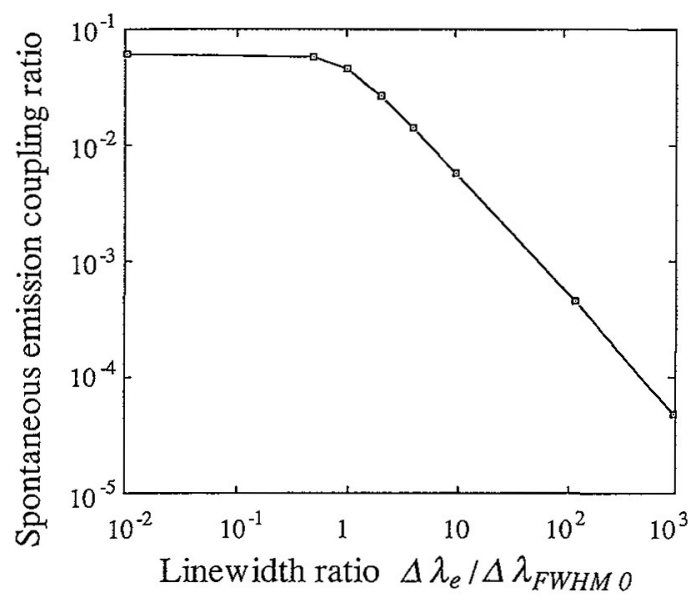

Fig. 4. The spontaneous emission coupling factor as a function of the relative linewidth $\Delta \lambda_{\text {em }} / \Delta \lambda_{\text {FwhMo. }}$. The cavity data are the same as in Fig. 1.

The spontaneous emission and lasing characteristic were investigated by optically pumping the microcavity at well above the bandgap in a liquid Helium cryostat. The fluorescence linewidth $\Delta \lambda_{\text {FWHM }}$ was $33 \mathrm{~nm}$ at room temperature and $2.5 \mathrm{~nm}$ at $4 \mathrm{~K}$. The reduced fluorescence linewidth at lower temperatures allows a much better match with the cavity resonance linewidth and an improved coupling of the spontaneous emission to the lasing mode. The cavity resonance linewidth was measured to be $\Delta \lambda_{\text {cavity }}=0.4 \mathrm{~nm}$ by observing the filtered fluorescence by a scanning FabryPerot interferometer. This corresponds to a photon lifetime of $\tau_{p}=0.8 \mathrm{psec}$ and a coherent mode radius of $a_{p}=3.8 \mu \mathrm{m}$ [14]. Looking at Fig. $4, \beta$ is estimated to be around $10^{-2}$ at $4 \mathrm{~K}$ and $5 \times 10^{-4}$ 
at $300 \mathrm{~K}$ if $\Delta \lambda_{\mathrm{FWHM}}=0.4 \mathrm{~nm}$ (direct measurement result) is taken. The measured input/output characteristic at $T=4 \mathrm{~K}$ is shown in Fig. 5. The pump light is focused to a spot of $25 \mu \mathrm{m}$ diameter. The output power was measured in a $0.75 \mathrm{~nm}$ resolution bandwidth centered around the lasing wavelength $811 \mathrm{~nm}$ by the monochromator and the photomultiplier. An absorbed threshold power defined at the point of the cavity internal photon number of $n=1$ was $85 \mu \mathrm{W}$ and absorbed threshold power density is $18 \mathrm{~W} / \mathrm{cm}^{2}$. The threshold pump power densities were found to be constant as far as the excitation spot size was larger than the (coherent) mode spot size of $7.6 \mu \mathrm{m}$. The single-mode threshold power was, therefore, estimated to be $7 \mu \mathrm{W}$ [14]. This value should be compared to the theoretical threshold pump power of $14 \mu \mathrm{W}$ calculated by $P_{t h}=\hbar \omega / 2 \beta \tau_{p}$ [14]. The solid line is a theoretical input/output curve for $\beta=10^{-2}$.

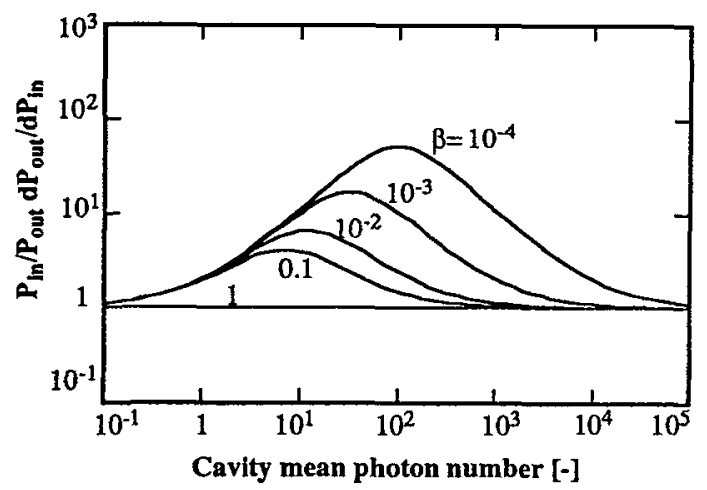

Fig. 6. (a) Theoretical differential increase of the differential quantum efficiency $\delta\left(\Delta I_{\text {out }} / \Delta I_{\text {in }}\right)$ vs. average photon number as a function of the spontaneous emission coupling efficiency $\beta$.

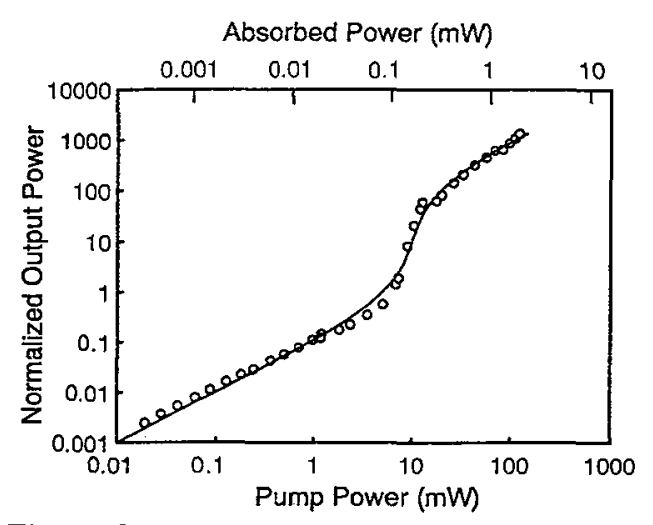

Fig. 5. Output power vs. input power for the planar structures. The solid lines are the theoretical curves. The vertical scales are normalized to the theoretical number of photons in the lasing mode, being unity at threshold.

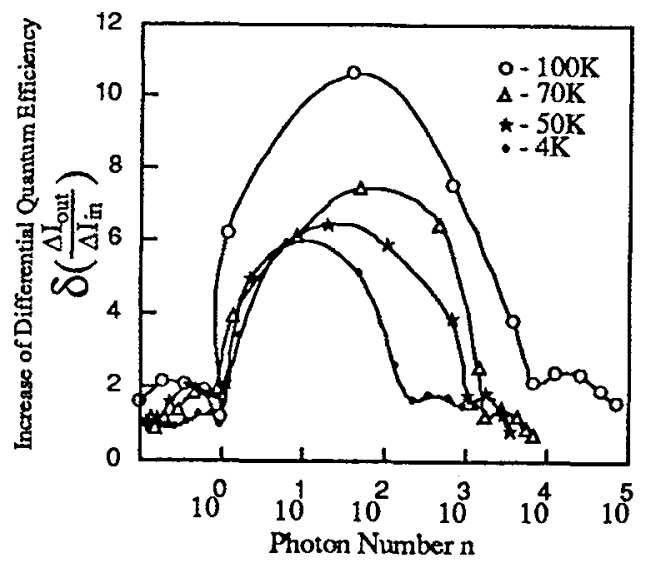

(b) Experimental differential increase of the differential quantum efficiency $\delta\left(\Delta I_{\text {out }} / \Delta I_{\text {in }}\right)$ vs. average photon number as a function of temperature.

In Figs. 6 (a) and (b), the theoretical and experimental increase of the differential quantum efficiency vs. the photon number are drawn for various temperatures. The values of $\beta$ were measured to be $10^{-2}$ at $T=4 \mathrm{~K}, 6 \times 10^{-3}$ at $T=50 \mathrm{~K}, 1.3 \times 10^{-3}$ at $T=70 \mathrm{~K}$, and $4.4 \times 10^{-4}$ at $T=100 \mathrm{~K}$. Those experimental results are well explained by the theoretical curve in Fig. 4.

The emission linewidth measured by the scanning Fabry-Perot cavity at $4 \mathrm{~K}$ is plotted as a function of a normalized pump rate in Fig. 7. The emission linewidth is equal to the cold cavity bandwidth $(\simeq 200 \mathrm{GHz}$ ) at below the threshold and decreases to $\sim 20 \mathrm{GHz}$ at above the threshold. A polarization characteristic of the emission is shown in Fig. 8. The emission has a completely random polarization at below the threshold, but a linear polarization at above the threshold. In the present case, the polarization direction was the same as the crystal direction (011) and the selection may be related to strain induced during epitaxial growth. 


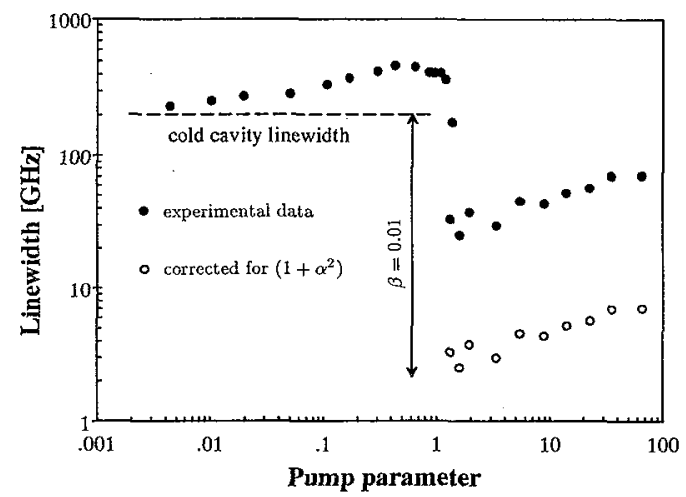

Fig. 7. Laser linewidth of the planar structure vs. pump power (normalized to unity at threshold).

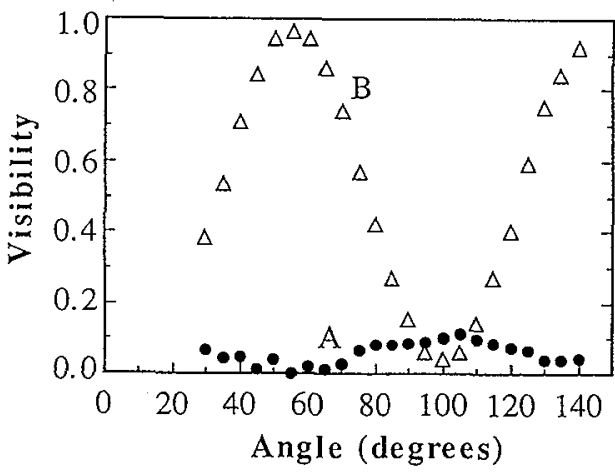

Fig. 8. Experimentally measured polarization characteristic: light power as a function of rotation angle of the Babinet-Soleil compensator. Curve $A$ is for low pump powers, curve $B$ for high pump powers.

\section{Coherent spontaneous emission with resonant optical pumping}

When cold excitons in the QW is optically excited by a resonant pump wave, coherent excitonic dipoles radiate collectively with the cavity enhanced vacuum fluctuations before they dephase or depolarize. We will discuss such coherent spontaneous emission process in this section.

Figure 9 shows the input/output characteristics at various temperatures under the cw resonant excitation. The same QW microcavity discussed in the previous section is optically pumped by a tunable Ti: $\mathrm{Al}_{2} \mathrm{O}_{3}$ laser with an incident angle of 2.5 to $5^{\circ}$. The coherent emission (or Rayleigh scattering [12][13]) from the sample in a normal direction is measured by the monochromator (wavelength filter) and the photomultiplier. At temperatures between $4 \mathrm{~K}$ and $40 \mathrm{~K}$, the coherent emission coupling efficiency into the cavity resonant mode was measured to be $100 \%$, that is, a so-called "thresholdless" behavior was observed. At temperatures above $50 \mathrm{~K}$, however, the coherent emission coupling efficiency decreased to below $100 \%$ at a low pump region and the threshold (the jump in a differential quantum efficiency from $\Delta P_{\text {out }} / \Delta P_{\text {in }}=\beta$ to $\Delta P_{\text {out }} / \Delta P_{\text {in }}=1$ ) was observed, where $\Delta P_{\text {out }}$ and $\Delta P_{\text {in }}$ are the incremental output coherent emission power and the input pump power. However, this threshold was not necessarily related to the cavity internal photon number $n=1$, as shown in Fig. 10. A coherent emission with $100 \%$ quantum efficiency was recovered when the cavity internal photon number reached $n \approx 10^{4}$.

The spectral linewidth of the coherent emission at $4 \mathrm{~K}$ was identical to that of the pump light, for instance, $\sim 2 \mathrm{GHz}$ for the Ti: $\mathrm{Al}_{2} \mathrm{O}_{3}$ laser pumping case and $\sim 30 \mathrm{MHz}$ for the GaAs semiconductor laser pumping case, and was totally independent of the pump rate. This is in sharp contrast to the spectral linewidth of the incoherent spontaneous emission discussed in the previous section. The polarization of the coherent emission was also identical to that of the pump light and was not effected by the crystal axis.

The coherent emission coupling efficiency into the cavity resonant mode was decreased if hot electron-hole pairs were simultaneously excited at well above the bandgap as shown in Fig. 11. In this experiment, the GaAs semiconductor laser at $811 \mathrm{~nm}$ resonantly excited cold excitons and the Ti: $\mathrm{Al}_{2} \mathrm{O}_{3}$ laser at $760 \mathrm{~nm}$ excited hot electron-hole pairs. When the free carrier density exceeds the level corresponding to one electron-hole pair per the coherence area of a cold exciton, the coherent emission efficiency started to decrease and the spectral linewidth started to increase. 


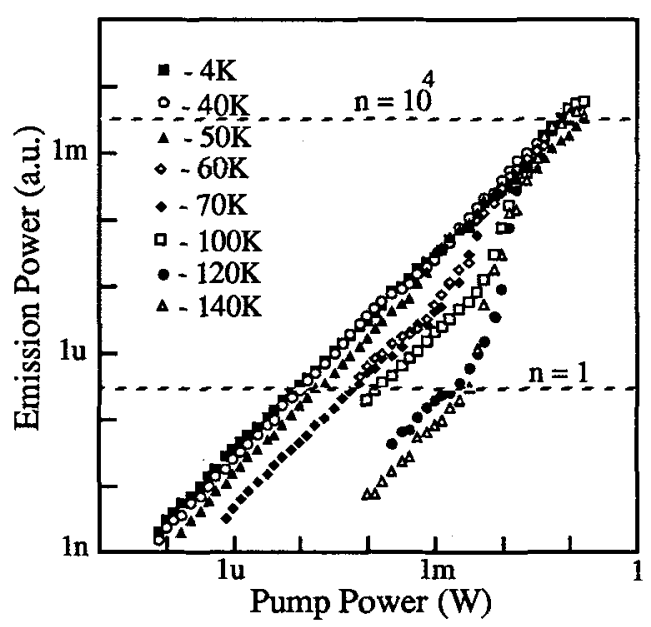

Fig. 9. The input/output characteristics at various temperatures under the $\mathrm{cw}$ resonant excitation.

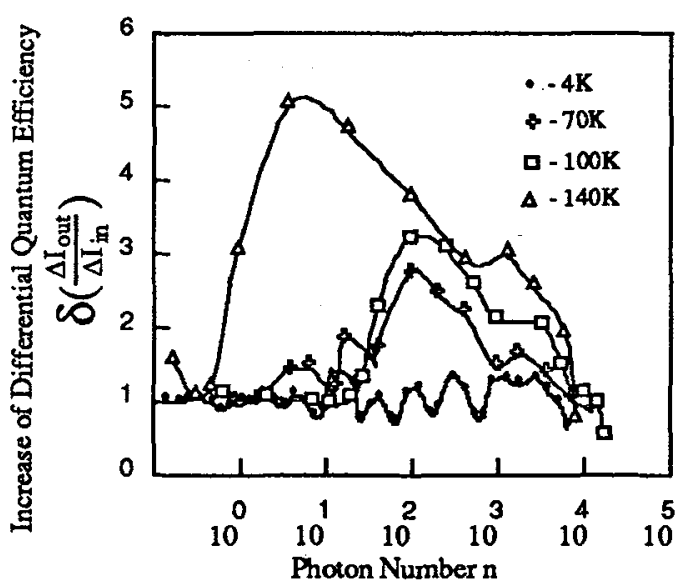

Fig. 10. The change in the differential quantum efficiency $\delta\left(\frac{\Delta P_{\text {out }}}{\Delta P_{\text {in }}}\right)$ vs. the photon number $n$ under the $\mathrm{cw}$ resonant excitation.

Those experimental results can be interpreted as follows. The scattering rate of the coherent exciton dipoles is enhanced either by increasing the temperature (exciton-phonon scattering) or creating the hot electron-hole pairs (excitoncarrier scattering). The dephasing of the coherent exciton dipoles due to such scattering was manifested by the increase of the emission linewidth. The thermalization of the coherent exciton dipoles due to such scattering was manifested by the decrease of the coherent emission intensity. The threshold behavior shown in Figs. 9 and 10 should not be the conventional lasing (onset of stimulated emission of photons) characterized by $n=1$ but may be a nonlinear locking behavior of an exciton-polariton. (The number of independent (coherent) excitons in the coherence area of the microcavity optical mode is about $10^{4}$.)

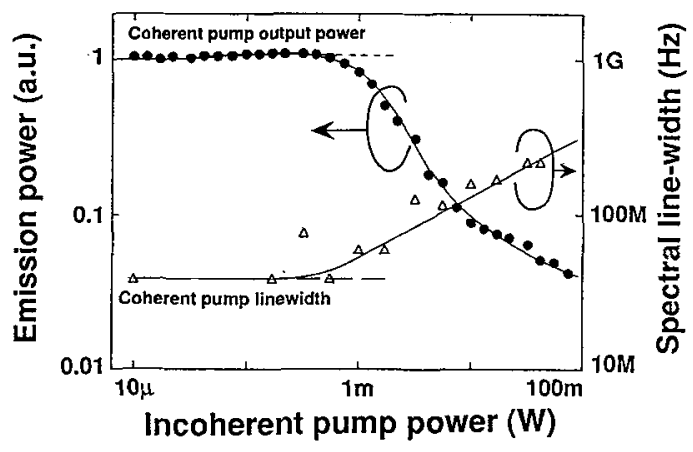

Fig. 11. The coherent emission intensity into the cavity resonant mode and the spectral linewidth vs. incoherent pumping power.

Figure 12 (a) shows a typical photoluminescence excitation (PLE) spectrum at $4 \mathrm{~K}$. The splitting of the PLE spectrum corresponds to the two exciton-polariton normal modes. The frequency seperation is calculated as [16]

$$
f_{\mathrm{split}}=\left(\frac{\alpha_{0} d_{Q W} \delta_{H} \Delta_{\mathrm{FSR}}}{\pi}-\frac{\left(\delta_{H}-\delta_{c}\right)^{2}}{4}\right)^{1 / 2},
$$

where $\alpha_{0} d_{Q W}$ is the peak absorption at the 1 s heavy hole exciton wavelength in the QW of thickness $d_{Q W}, \delta_{H}$ is the homogeneous linewidth of the $1 \mathrm{~s}$ heavy hole exciton determined by the dephasing time, $\delta_{c}$ is the cavity linewidth, and $\Delta_{\mathrm{FSR}}=c_{0} / 2 n L_{c}$ is the cavity free spectral range. As shown in Fig. 12 (b), the normal mode splitting decreases with increasing the homogeneous linewidth $\delta_{H}$. Experimentally, we observed that the normal mode splitting was $\sim 0.15 \mathrm{~nm}$ at $4 \mathrm{~K}$, decreasing with increasing a temperature and disappeared at $\sim 50 \mathrm{~K}$. This result can be interpreted as the increase of 
the dephasing scattering. A similar decrease in the normal mode splitting was observed by increasing the pump rate. This result is compatible with the theoretical prediction for the transition from the two-peak vacuum Rabi splitting to the three-peak ac Stark splitting (Mollow's triplet) by the increase of the coherent radiation field in the cavity [17].

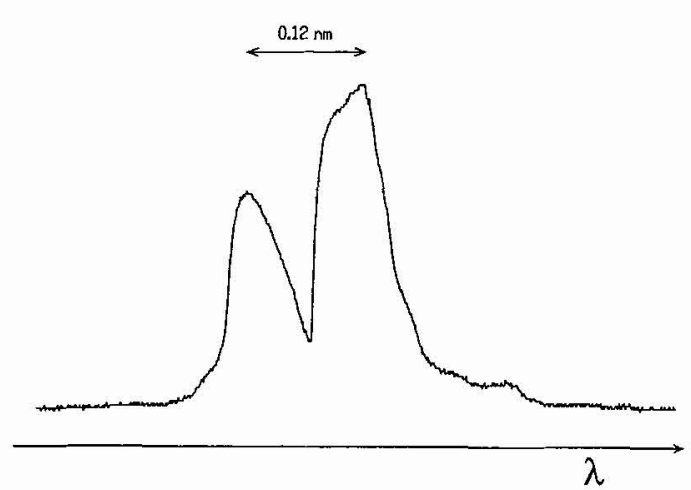

Fig. 12. (a) The observed exciton-polariton normal mode splitting.

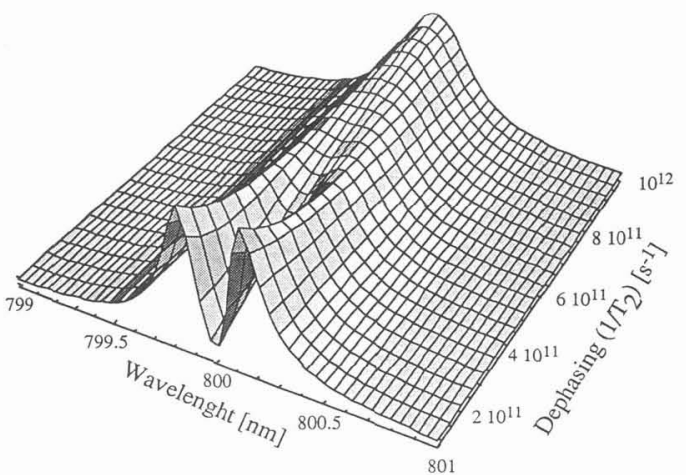

(b) The theoretical normal mode splitting $f_{\text {split }}$ vs. the homogeneous linewidth $\delta_{H}$.

\section{Transient response with pulse excitation}

When the QW was excited at $4 \mathrm{~K}$ by the two successive 100 fsec pulses from the $\mathrm{Ti}: \mathrm{Al}_{2} \mathrm{O}_{3}$ laser at well above the bandgap, we observed the seperation time-dependent spontaneous emission intensity as shown in Fig. 13. The first pulse excites the QW just below the threshold and the second pulse turns on lasing if the second pulse arrives before the population decays radiatively. However, if the second pulse arrives after the population decays radiatively, the second pulse does not turn on lasing. In this way, the measured decay time of about $200 \mathrm{psec}$ is considered as the radiative lifetime of incoherent spontaneous emission at $4 \mathrm{~K}$.
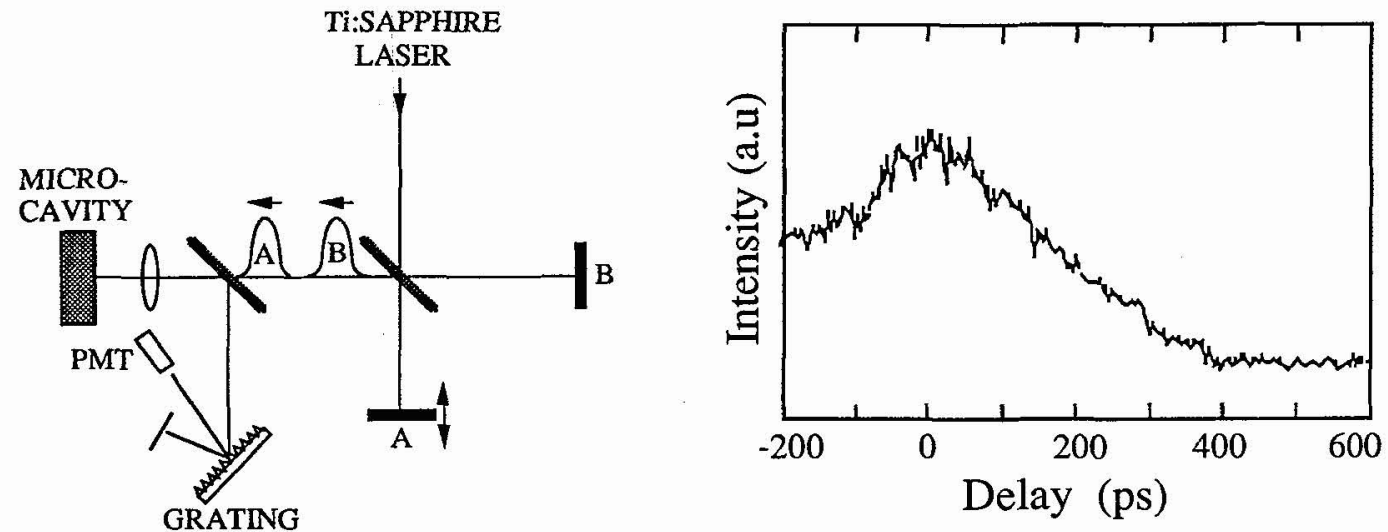

Fig. 13. Time delay incoherent spontaneous emission measurement for excitation with two successive nonresonant 100 fsec pulses. (a) Experimental setup. (b) Measurement result.

Figure 14 shows the result of the cross-polarized pump-probe experiment of the resonant excitation of cold excitons at $4 \mathrm{~K}$. The pump and variably delayed probe pulses are in orthogonal polarizations. A coherent Rabi oscillation with a 650 fsec period, damped by a cavity photon lifetime of 0.8 psec was clearly seen in the differential reflection coefficient of the probe pulse.

One of the authors (Y. Y.) would like to thank E. Hanamura, D. Chemla, A. Mysyrowicz, R. 
Slusher and F. DeMartini for their critical discussions.
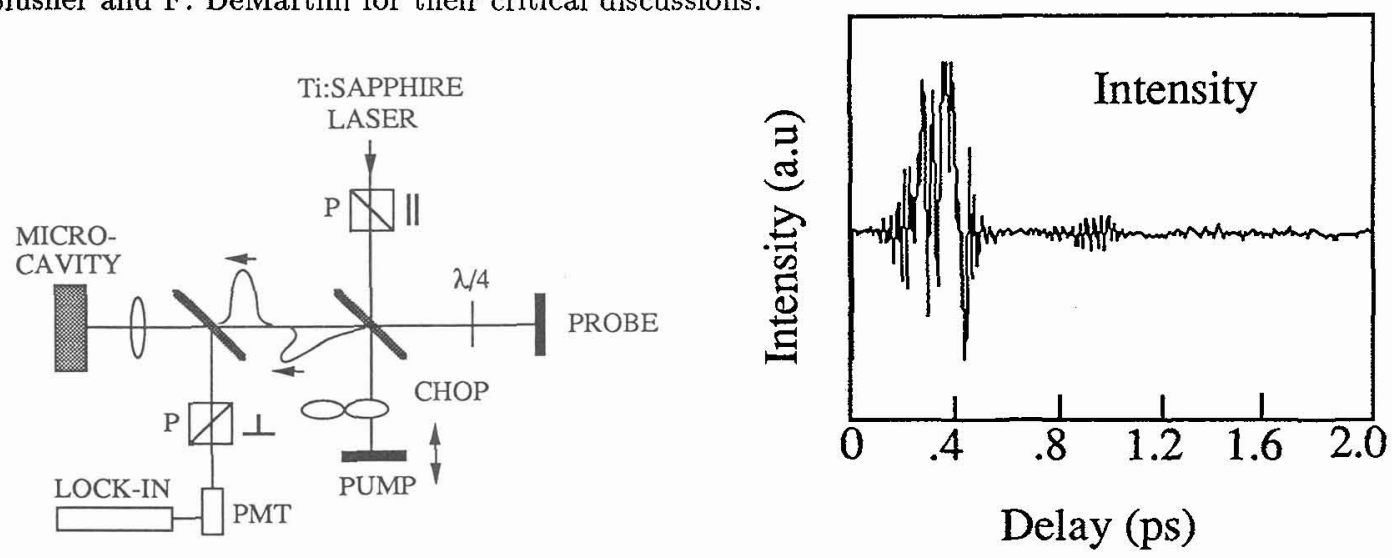

Fig. 14. Cross-polarization pump-probe measurement for resonant excitation. (a) Experimental setup. (b) Measured amplitude of the reflected probe pulse vs. delay.

\section{References}

[1] Purcell, E. M., Phys. Rev. 69 (1946) 681.

[2] Casimir H. B. and Polder D., Phys. Rev., 73 (1948) 360.

[3] Jaynes E. T. and Cummings J. F., Proc. IEEE, 51 (1963) 89.

[4] Walther H., Phys. Rep., 219 (1992) 263; Meystre P. ibid 219 (1992) 243.

[5] Haroche S. and Kleppner D., Physics Today 42 (1989) 24.

[6] Morin S. E., Wu Q. and Mossberg T. W., Optics and Photonics News 3 (1992) 10.

[7] Yamamoto Y., Machida S., Igeta K. and Horikoshi Y., in Coherence and Quantum Optics VI, J. H. Eberly et al. eds., Plenum, New York (1989) 1249; Yamamoto Y. and Slusher R. E., Physics Today 46 (1993) 66.

[8] Weisbuch C., Nishioka M., Ishikawa A. and Arakawa Y., Phys. Rev. Lett., 69 (1992) 3314.

[9] Hopfield J. J., Phys. Rev. 112 (1958) 1555.

[10] Andreani, L. C., Tassone F. and Bassani F., Solid State Commun. 77 (1991) 641.

[11] Deveaud B., Clerot F., Roy N., Stazke K., Sermage B. and Katzer D. S., Phys. Rev. Lett., 67 (1991) 2355.

[12] Hegarty J., Sturge M. D., Weisbuch C., Gossard A. C. and Wiegmann W., Phys. Rev. Lett., 49 (1982) 930 .

[13] Stoltz H., Schwarze D., von der Osten W., and Weimann G., Superlatt. and Microstruct., 9 (1991) 511.

[14] Björk, G., Heitmann H. and Yamamoto Y., Phys. Rev A. 47 (1993) 4451.

[15] Yamamoto Y., Machida S. and Björk G., in Coherence, Amplification and Quantum Effects in Semiconductor lasers, Wiley, New York (1990).

[16] Zhu, Y., Gauthier D. J., Morin S. E., Wu Q., Carmichael H. J. and Mossberg T. W., Phys. Rev. Lett. 64 (1991) 2499.

[17] Sanchez-Mondragon J. J., Narozhny N. B. and Eberly J. H., Phys. Rev. Lett. 51 (1983) 550. 